\title{
High gain harmonic generation free electron lasers enhanced by pseudoenergy bands
}

\author{
Takashi Tanaka* and Ryota Kinjo \\ RIKEN SPring-8 Center, Koto 1-1-1, Sayo, Hyogo 679-5148, Japan
}

(Received 15 March 2017; published 24 August 2017)

\begin{abstract}
We propose a new scheme for high gain harmonic generation free electron lasers (HGHG FELs), which is seeded by a pair of intersecting laser beams to interact with an electron beam in a modulator undulator located in a dispersive section. The interference of the laser beams gives rise to a two-dimensional modulation in the energy-time phase space because of a strong correlation between the electron energy and the position in the direction of dispersion. This eventually forms pseudoenergy bands in the electron beam, which result in efficient harmonic generation in HGHG FELs in a similar manner to the well-known scheme using the echo effects. The advantage of the proposed scheme is that the beam quality is less deteriorated than in other existing schemes.
\end{abstract}

DOI: 10.1103/PhysRevAccelBeams.20.080706

\section{INTRODUCTION}

Free electron lasers (FELs) have now become a promising scheme to produce high-power coherent light in short wavelength regions which are not accessible with conventional lasers. In spectral regions where reflective mirrors are not available, FELs currently under operation are divided into two types based on different principles: self-amplified spontaneous emission [1] and high gain harmonic generation (HGHG) [2]. In the former scheme, which is the basis of two running x-ray FEL facilities, LCLS [3] and SACLA [4], spontaneous radiation is amplified. In the latter scheme, which is the basis of FERMI [5], coherent seed light, or seed laser, is up-converted. Although the latter has many advantages against the former, its attainable wavelength is technically limited by two factors: wavelength $\left(\lambda_{s}\right)$ of the available seed laser, and up-conversion efficiency of the HGHG process.

Shortening $\lambda_{s}$ has been explored for many years [6-8]; even so, that of the seed laser currently available in HGHG FELs is in the spectral region from infrared to ultraviolet, mainly because it should be sufficiently stable to be spatiotemporally synchronized with the electron beam. It is thus the recent trend to improve the up-conversion efficiency to realize HGHG FELs in shorter wavelengths, as addressed in the following.

The up-conversion in HGHG FELs is achieved by upconverting the microbunch formed in the electron beam through interaction with the seed laser, which makes it

\footnotetext{
"ztanaka@spring8.or.jp
}

Published by the American Physical Society under the terms of the Creative Commons Attribution 4.0 International license. Further distribution of this work must maintain attribution to the author(s) and the published article's title, journal citation, and DOI. possible to generate coherent light having the wavelength of $\lambda_{s} / n$, where $n$ is an integer referred to as a harmonic order. It is well known that the electron beam should be strongly modulated in energy with an amplitude $\gamma_{M}$ larger than or comparable to $n \sigma_{\gamma}$, where $\sigma_{\gamma}$ is the rms energy spread of the electron beam [2]; this requirement poses two issues in HGHG FELs to aim at higher $n$. First, the required power of the seed laser, which is roughly proportional to $\gamma_{M}^{2}$, increases as $n^{2}$. Second, which may be more critical, larger $\gamma_{M}$ eventually deteriorates the electron beam quality and thus limits the achievable FEL performances [9].

To overcome the above difficulty in HGHG FELs, two different schemes based on special methods for energy modulation have been proposed, which are referred to as the echo-enabled harmonic generation (EEHG) [10-12] and the phase-merging enhanced harmonic generation (PEHG) $[13,14]$. The electron beam is modulated twice in the former, while the modulation is applied in a dispersive section in the latter; both of them significantly relax the above requirement on the energy modulation and thus the up-conversion efficiency is much better than what is expected in the original HGHG scheme. As a result, the performances of EEHG and PEHG schemes are expected to be much better than that of the normal HGHG scheme.

An attractive application of these schemes is to generate coherent radiation in storage rings (SRs) especially in short-wavelength regions [15,16], which significantly enhances the achievable brightness of existing and upcoming SR facilities. As discussed later in detail, however, the quality of the electron beam can be significantly deteriorated in these schemes once it is modulated by the seed laser. This effectively limits the achievable performances in SRs where the electron beam should be repeatedly used. In this paper, we propose a new scheme for HGHG FELs, which can retard the quality deterioration of the electron beam, and thus is well compatible with the SR operation. 


\section{PRINCIPLE OF OPERATION}

Figure 1 shows a schematic layout of the proposed scheme, with the coordinate system to be used in the following discussions. It is composed of three undulators similar to the EEHG scheme, which are referred to as the first modulator, second modulator, and radiator. The difference is that the first modulator is located in a dispersive section, and a pair of lasers intersecting at an angle of $2 \alpha$ work as the seed laser.

Let us first consider the energy exchange between the electron beam and a pair of intersecting laser beams (ILBs) in the first modulator. The electric field of the ILBs is given as

$$
E(x, z, s)=2 E_{0} \cos \left(\alpha k_{s} x+\phi / 2\right) \mathrm{e}^{i\left(k_{s} s-k_{u} z\right)},
$$

with $k_{s}=2 \pi / \lambda_{s}$ and $k_{u}=k\left(c / \bar{v}_{z}-\cos \alpha\right)$, where $s$ is the relative longitudinal position with respect to the electron beam center, $\bar{v}_{z}$ is the average longitudinal velocity of the electron beam moving in the modulator, $c$ is the speed of light, $E_{0}$ is the field amplitude of the seed laser, and $\phi$ is the relative phase between the ILBs and assumed to be $\pi$ in the following discussion. The energy modulation $\delta \gamma$ given in the electron beam is then given by

$$
\delta \gamma(x, s)=\gamma_{M} \sin \left(\alpha k_{s} x\right) \sin \left(k_{s} s\right),
$$

where $\gamma_{M}$ depends linearly on $E_{0}$ and the second factor means that the energy modulation is a function of $x$ as well as $s$, which comes from the interference of the ILBs. To facilitate the following discussion, we introduce a variable $\eta=\gamma / \gamma_{0}-1$, where $\gamma$ is the Lorentz factor of an electron and $\gamma_{0}$ denotes its average over the electron beam.
We now discuss the motion of electrons in the $(x, \eta)$ phase space while they travel through the dispersive section with the strength $D$, where the first modulator is located. To be specific, we consider the electron distributions at four different positions (1)-(4) indicated in Fig. 1. We assume an electron initially positioned at $x=0$ and $\eta=\eta^{\prime}$ before entering the dispersive section, and define $x_{m}$ and $\eta_{m}$ as the coordinates at the $m$ th position. Then it is easy to show

$$
\begin{aligned}
& x_{1}=0, \quad x_{2}=x_{3}=\eta^{\prime} D, \\
& x_{4}=-\eta_{M} D \sin \left(\pi \eta^{\prime} / 2 \eta_{M 0}\right) \sin \left(k_{s} s\right),
\end{aligned}
$$

and

$$
\begin{aligned}
& \eta_{1}=\eta_{2}=\eta^{\prime}, \\
& \eta_{3}=\eta_{4}=\eta^{\prime}+\eta_{M} \sin \left(\pi \eta^{\prime} / 2 \eta_{M 0}\right) \sin \left(k_{s} s\right),
\end{aligned}
$$

with $\eta_{M}=\gamma_{M} / \gamma_{0}$ and $\eta_{M 0}=\pi / 2 \alpha k_{s} D$.

The evolution of the electron distribution mathematically given above is illustrated in Fig. 2 in two particular cases of $s= \pm \lambda_{s} / 4$, where the numbers correspond to respective longitudinal positions. Before the electron beam enters the first modulator, no difference is found between the two cases as shown in Figs. 2-(1) and 2-(2). What is important is that $\eta_{M}$ should be chosen so that the electron distribution in the $(x, \eta)$ phase space becomes similar to what is illustrated in Fig. 2-(3). Then the electrons after being modulated by the ILBs are more populated around the energies indicated by arrows in Fig. 2-(4). In other words, the energy distribution of the electron beam is quantized at discrete levels separated by an energy interval of $\Delta \eta=2 \eta_{M 0}$.

It is obvious that the above effects can be more or less smeared under realistic conditions, especially by the beam size in the direction of dispersion, and the energy

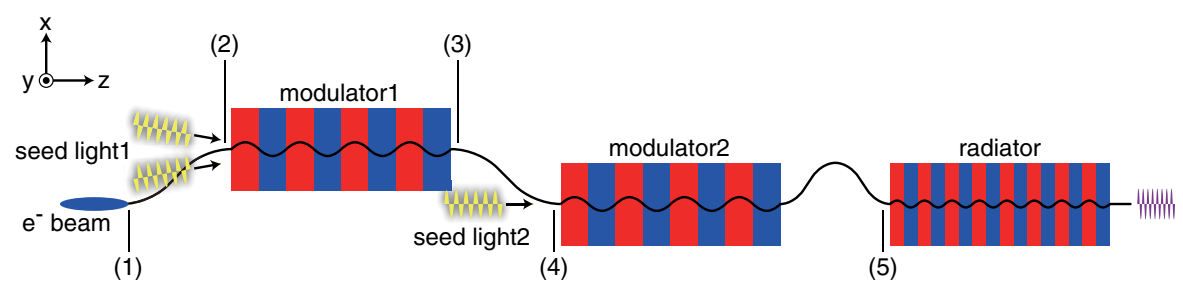

FIG. 1. Layout of the proposed scheme; the numbers from (1) to (5) show the longitudinal positions where the electron distributions are shown later in Fig. 2 or in Fig. 5.
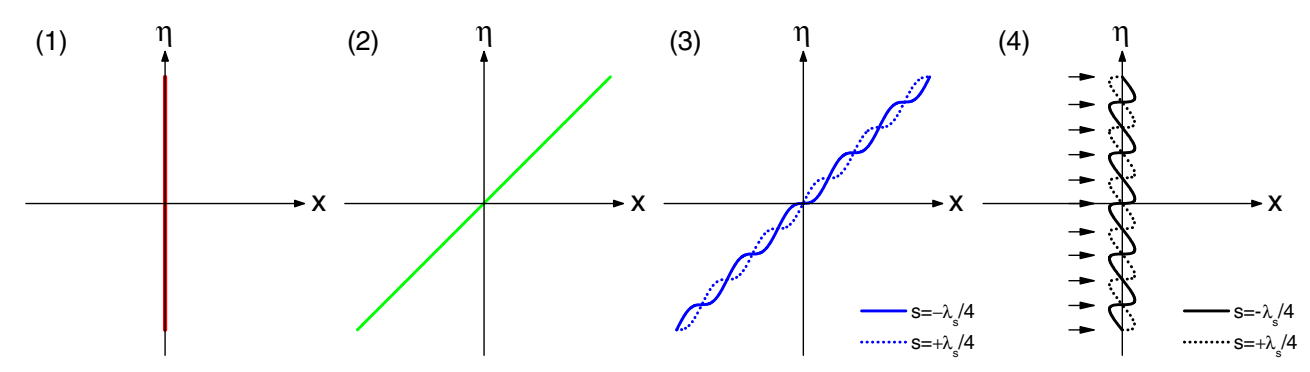

FIG. 2. Electron distributions in the phase space at four different positions indicated in Fig. 1. The solid and dashed lines in (3) and (4) correspond to $s=+\lambda_{s} / 4$ and $s=-\lambda_{s} / 4$, respectively. 

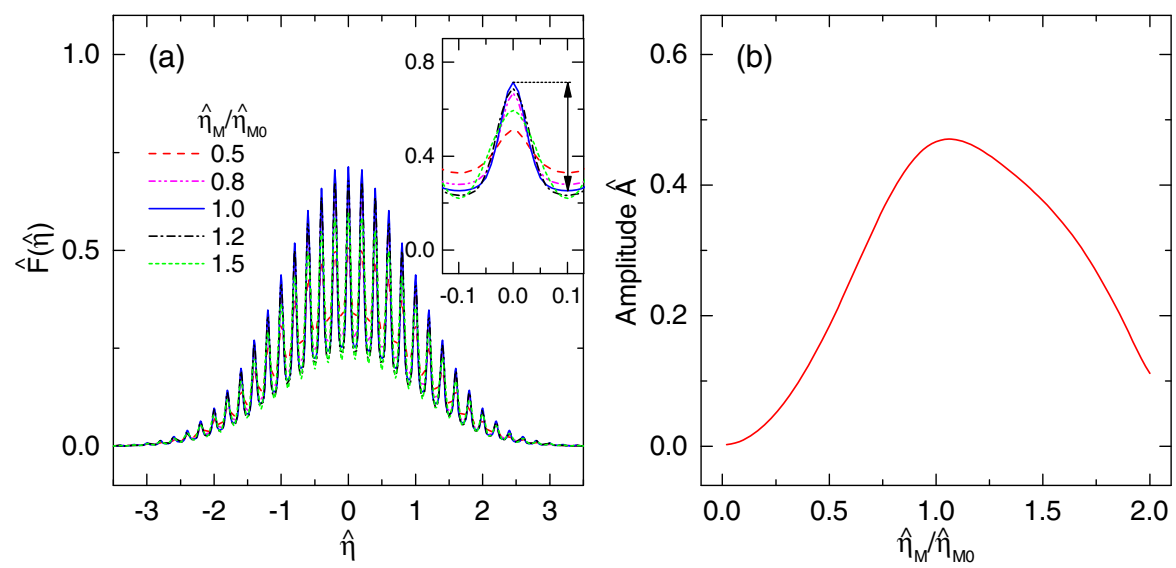

FIG. 3. Plots of the energy distribution function $\hat{F}$ computed with $\hat{\eta}_{M 0}=0.1$ and $\hat{\sigma}_{x}=0.02$ : (a) $\hat{F}(\hat{\eta})$ plotted as a function of $\hat{\eta}$ for different values of $\hat{\eta}_{M}$, and (b) $\hat{A}=\hat{F}(0)-\hat{F}\left(\hat{\eta}_{M 0}\right)$ plotted as a function of $\hat{\eta}_{M} / \hat{\eta}_{M 0}$. The arrow indicated in the inset of (a) shows $\hat{A}$ when $\hat{\eta}_{M} / \hat{\eta}_{M 0}=1$.

levels will transform to energy bands. In practice, such a structure should be referred to as "pseudoenergy bands," because it has nothing to do with quantum mechanics. If the beam size is too large, the band structure can completely disappear and the original distribution function is restored. To quantify its impact, let us evaluate the energy distribution function $F(\eta)$ at the position (4), assuming that the energy and horizontal distribution functions of the electron beam are given by Gaussian functions with the standard deviations of $\sigma_{\eta}$ and $\sigma_{x}$. In the same manner as described above, the coordinate of an electron initially positioned at $\left(x^{\prime}, \eta^{\prime}\right)$ is converted as $x_{4}=x^{\prime}+\left(\eta^{\prime}-\eta_{4}\right) D$ and $\eta_{4}=\eta^{\prime}+\eta_{M} \sin \left[\left(\pi / 2 \eta_{M 0}\right)\left(x^{\prime} / D+\eta^{\prime}\right)\right] \sin \left(k_{s} s\right)$. Then we have

$$
\begin{aligned}
\hat{F}(\hat{\eta})= & \frac{1}{2 \pi^{2} \hat{\sigma}_{x}} \int_{-\pi / 2}^{\pi / 2} d \psi \int_{-\infty}^{\infty} d \hat{x} \\
& \times \exp \left\{-\frac{[\hat{x}-H(\hat{\eta}, \hat{x}, \psi)]^{2}}{2 \hat{\sigma}_{x}^{2}}-\frac{H^{2}(\hat{\eta}, \hat{x}, \psi)}{2}\right\},
\end{aligned}
$$

with

$$
H(\hat{\eta}, \hat{x}, \psi)=\hat{\eta}+\hat{\eta}_{M} \sin \psi \sin \left(\pi \hat{x} / 2 \hat{\eta}_{M 0}\right),
$$

where we have introduced normalized variables defined by $\hat{x}=x / \sigma_{\eta} D, \hat{\eta}=\eta / \sigma_{\eta}, \hat{\sigma}_{x}=\sigma_{x} / \sigma_{\eta} D, \hat{\eta}_{M}=\eta_{M} / \sigma_{\eta}, \hat{\eta}_{M 0}=$ $\eta_{M 0} / \sigma_{\eta}, \hat{F}=\sigma_{\eta} F$, together with the electron phase $\psi=k_{s} s$.

Figure 3(a) shows the plot of $\hat{F}(\hat{\eta})$ computed with $\hat{\eta}_{M 0}=0.1$ and $\hat{\sigma}_{x}=0.02$, for different values of $\hat{\eta}_{M}$, with the inset showing the detail around $\hat{\eta}=0$. A number of peaks, or more specifically energy bands, are found as expected, and they become most distinct when $\hat{\eta}_{M}=\hat{\eta}_{M 0}$. This is a criterion that the ILBs should satisfy to form the energy bands in the electron beam; we note, however, that this criterion is not so stringent. This is obvious from Fig. 3(b), where the maximum amplitude of $\hat{F}$ defined as $\hat{A}=\hat{F}(0)-\hat{F}\left(\hat{\eta}_{M 0}\right)$ is plotted as a function of $\hat{\eta}_{M} / \hat{\eta}_{M 0}$.
For reference, $\hat{A}$ for $\hat{\eta}_{M} / \hat{\eta}_{M 0}=1$ is indicated by an arrow in the inset of Fig. 3(a). Roughly speaking, a 10\% variation of $\hat{\eta}_{M}$ from the optimum value just amounts to a $1 \%$ reduction of $\hat{A}$. This means that a $20 \%$ variation of the power density of the ILBs has a negligible impact on the formation of the energy bands.

Figure 4(a) shows the sensitivity of the energyband structure to $\hat{\sigma}_{x}$, where $\hat{F}(\hat{\eta})$ is computed with $\hat{\eta}_{M}=\hat{\eta}_{M 0}=0.1$ for different values of $\hat{\sigma}_{x}$. For example, $\hat{F}(\hat{\eta})$ reduces to the original Gaussian function when $\hat{\sigma}_{x}=0.1$. It is thus important to have a smaller beam size, or a smaller emittance, in the direction of dispersion to generate a more distinct energy-band structure.

We note that $10\left(=\hat{\eta}_{M 0}^{-1}\right)$ energy bands are found within the region of $|\hat{\eta}| \leq 1$; this is obvious from the fact that the energy-band interval is given as $2 \eta_{M 0}$. In this regard, it is interesting to decrease the number of energy bands by applying larger $\hat{\eta}_{M 0}$. For example, $\hat{F}(\hat{\eta})$ for different values of $\hat{\sigma}_{x}$ with $\hat{\eta}_{M 0}=2$ are plotted in Fig. 4(b), where the only energy band appears around $\hat{\eta}=0$ with a higher peak intensity, meaning that the energy spread of the electron beam is effectively reduced. This suggests another possibility of the proposed scheme; for example, it can be applied to cooling, or more precisely, emittance exchange of an electron beam having a large energy spread, such as that generated in the laser wakefield accelerator [17].

After the energy bands are formed in the electron beam in the first modulator, it further undergoes an energy modulation in the second modulator, which is then converted to a density modulation in the following chicane with the momentum compaction of $R_{56}$. To discuss the modulation there, we need a mathematical expression for the distribution function in the $(s, \eta)$ phase space, $G_{4}(s, \eta)$, at the position (4) indicated in Fig. 1, i.e., just before the second modulator. Recalling that the energy-band interval is $2 \eta_{M 0}$, it is reasonable to assume 

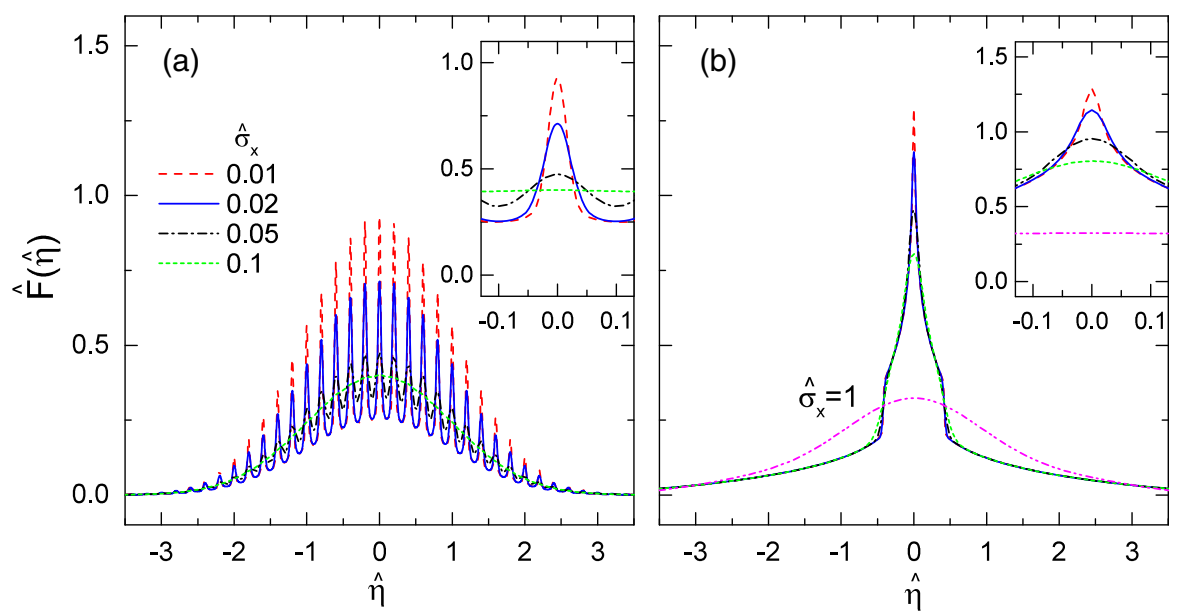

FIG. 4. Same as Fig. 3(a), but with (a) $\hat{\eta}_{M 0}=0.1$ and (b) $\hat{\eta}_{M 0}=2$. In both cases, $\hat{\eta}_{M}=\hat{\eta}_{M 0}$.

$G_{4}(s, \eta)=\frac{1}{\sqrt{2 \pi} \sigma_{\eta}} \exp \left(-\frac{\eta^{2}}{2 \sigma_{\eta}^{2}}\right)\left[1+\sum_{m=1} \delta_{F, m} \cos \left(\frac{m \pi \eta}{\eta_{M 0}}\right)\right]$,

where $m$ denotes the harmonic order of the energy-band structure having the period of $2 \eta_{M 0}$, and $\delta_{F, m}$ denotes its amplitude. Note that $G_{4}(s, \eta)$ does not actually include the variable $s$, meaning that no density modulation is present after passing through the dispersion section because of the large momentum compaction.

The distribution function $G_{5}(s, \eta)$ at the position (5) indicated in Fig. 1, after the electron beam passes through the second modulator and the chicane, is given as

$$
G_{5}(s, \eta)=G_{4}\left\{s, \eta-\eta_{H} \sin \left[k_{s}\left(s-R_{56} \eta\right)\right]\right\}
$$

where $\eta_{H}$ is the energy modulation amplitude given in the second modulator.

The bunching factor $b_{n}$ at the $n$th harmonic is then given by integrating $G_{5}$ over $\eta$ and expanding into a Fourier series. As in the same manner derived in [2], we have

$b_{n} \equiv \frac{1}{\lambda_{s}} \int_{-\lambda_{s} / 2}^{\lambda_{s} / 2} \mathrm{e}^{i n k_{s} s} d s \int_{-\infty}^{\infty} d \eta G_{5}(s, \eta)=b_{1 n}+b_{2 n}$

with

$$
\begin{gathered}
b_{1 n}=J_{n}\left(n \rho \hat{\eta}_{H}\right) \exp \left(-n^{2} \rho^{2} / 2\right), \\
b_{2 n}=\sum_{m=1} b_{2 n, m},
\end{gathered}
$$

and

$$
\begin{aligned}
b_{2 n, m}= & \frac{\delta_{F, m} J_{n}\left(n \rho \hat{\eta}_{H}\right)}{2}\left\{\exp \left[-\frac{1}{2}\left(n \rho+\frac{m \pi}{\hat{\eta}_{M 0}}\right)^{2}\right]\right. \\
& \left.+\exp \left[-\frac{1}{2}\left(n \rho-\frac{m \pi}{\hat{\eta}_{M 0}}\right)^{2}\right]\right\},
\end{aligned}
$$

where $J_{n}$ is the $n$th order Bessel function of the first kind, and $\rho=k_{s} R_{56} \sigma_{\eta}$ is the normalized momentum compaction of the chicane. The first term $b_{1 n}$ denotes the bunching factor due to the normal HGHG process exponentially decaying as $n^{2}$, while the second term comes from the energy-band structure in the electron beam. If $\rho=\pi / n \hat{\eta}_{M 0}$ is satisfied, only the fundamental term survives to give

$$
b_{2 n} \sim \frac{\delta_{F, 1}}{2} J_{n}\left(\pi \frac{\hat{\eta}_{H}}{\hat{\eta}_{M 0}}\right) .
$$

Recalling that the maximum value of $J_{n}$ scales as $n^{-1 / 3}$, it is obvious that $b_{2 n}$ decays as $n$ much more slowly than $b_{1 n}$, if relevant parameters are optimized to satisfy $\rho=\pi / n \hat{\eta}_{M 0}$. This suggests that a much larger harmonic order is available in the HGHG FELs based on the electron beam with the energy-band structure.

It should be mentioned here that the energy modulation in the dispersive section, which is one of the essential processes in the proposed scheme, gives rise to an increase in the beam size and thus the emittance growth in the direction of dispersion as is evident from Fig. 2-(4). To be specific, the emittance grows by a factor of $\hat{\varepsilon}=\sqrt{1+\left(\sigma_{M} / \sigma_{x}\right)^{2}}$, with

$$
\sigma_{M}^{2}=\left\langle\int_{-\infty}^{\infty} x^{2} \delta\left(x-x_{4}\right) d x\right\rangle=\left(\frac{D \eta_{M}}{2}\right)^{2},
$$

where $\delta$ is the Dirac delta function and $\langle\cdots\rangle$ means an average with respect to $s$ and $\eta^{\prime}$. Substituting the normalized variables, we have $\hat{\varepsilon}=\sqrt{1+\left(\hat{\eta_{M}} / 2 \hat{\sigma_{x}}\right)^{2}}$. For example, $\hat{\varepsilon}$ amounts to 1.4 in the condition of $\hat{\eta}_{M}=0.1$ and $\hat{\sigma}_{x}=0.05$ as shown in Fig. 4(a). 


\section{EXAMPLE}

To illustrate a possible performance of the proposed scheme, we need to carry out FEL simulations with realistic electron beam parameters. Here, we assume an electron beam in a SR, instead of a linear accelerator, because it is more compatible with the proposed scheme in terms of a relatively large energy spread and small emittance in the vertical direction. Furthermore, realization of SR-based FELs in short-wavelength regions is apparently attractive in terms of enhancing the peak and average brightness of the SR-based facilities. It should be noted, however, that this does not exclude the possibility of applying the proposed scheme to XFEL facilities based on linear accelerators, albeit we do not go into details in this paper.

As an example, we assume a SR with the circumference of $200 \mathrm{~m}$, which stores a single-bunch electron beam with the energy of $1 \mathrm{GeV}$, natural emittance of $10 \mathrm{~nm} \mathrm{rad}$, coupling constant of $0.5 \%$, energy spread of $0.1 \%$, bunch charge of $13 \mathrm{nC}$, and FWHM bunch length of $50 \mathrm{ps}$. The resultant peak and average beam currents are 250 and $20 \mathrm{~mA}$, respectively. Although there are no SRs currently running with these parameters, they are feasible enough to be achieved with the state-of-the-art accelerator theories and technologies.

We also assume that the seed laser with $\lambda_{s}=267 \mathrm{~nm}$ modulates the electron beam to emit coherent radiation at the 20th harmonic $(13.4 \mathrm{~nm})$. The first modulator with the period length of $40 \mathrm{~mm}$ and peak magnetic field of $1.02 \mathrm{~T}$ is located in a vertical dispersive section with $D=0.2 \mathrm{~m}$, where ILBs with $\alpha=3.34 \mathrm{mrad}$ and the power density of $5.0 \times 10^{8} \mathrm{~W} / \mathrm{cm}^{2}$ are injected synchronously with the electron beam. The second modulator has the period length of $60 \mathrm{~mm}$ and peak field of $1.45 \mathrm{~T}$, where the electron beam is further modulated by a single laser beam (not ILB) with the power density of $7.9 \times 10^{9} \mathrm{~W} / \mathrm{cm}^{2}$. Then, the energy modulation is converted to the density modulation in a chicane with $R_{56}=0.056 \mathrm{~mm}$, and the electron beam emits coherent radiation at $13.4 \mathrm{~nm}$ in the radiator with the period length of $24 \mathrm{~mm}$ and peak field of $1.14 \mathrm{~T}$. Note that the (first and second) modulators and radiator are $2 \mathrm{~m}$ long, and the horizontal and vertical betatron functions are 2 and $1 \mathrm{~m}$ at the center of each of them, respectively. It is relevant to mention that $\hat{\eta}_{M} \sim \hat{\eta}_{M 0} \sim 0.1$ is satisfied with the above parameters, and thus energy bands similar to those shown in Fig. 4(a) are expected.

Note that the time-averaged brightness (given in photons $/ \mathrm{sec} / \mathrm{mm}^{2} / \mathrm{mrad}^{2} / 0.1 \%$ b.w.) of spontaneous radiation at the wavelength of $13.4 \mathrm{~nm}$, which is emitted by the electron beam moving in the radiator, is estimated as $1.9 \times 10^{18}$, with a numerical code SPECTRA [18] using the above parameters. Considering the peak current of $250 \mathrm{~A}$ and the average current of $20 \mathrm{~mA}$, this can be converted to the peak brightness of $2.4 \times 10^{22}$.

The FEL simulations have been carried out using the simulation code SIMPLEX [19] through the whole system from the first modulator to the radiator, in which tracking of the macroparticles in the six-dimensional phase space is carried out and thus the emittance increase effect is taken into account. Note that only a limited region around the bunch center having the peak current of $250 \mathrm{~A}$ has been simulated instead of the whole electron bunch, which is enough to evaluate the expected performance of the proposed scheme.

Figures 5(a)-5(c) show the distributions of macroparticles retrieved from the simulation results after the first modulator [at the position (3) in Fig. 1], before the second modulator (4), and before the radiator (5), respectively. The "weblike pattern" found in (a) is specific to the modulation by the ILBs, which reduces to the energy bands as found in (b), after the large momentum compaction is applied in the dispersive section. After passing through the second modulator and the chicane, each energy band is independently
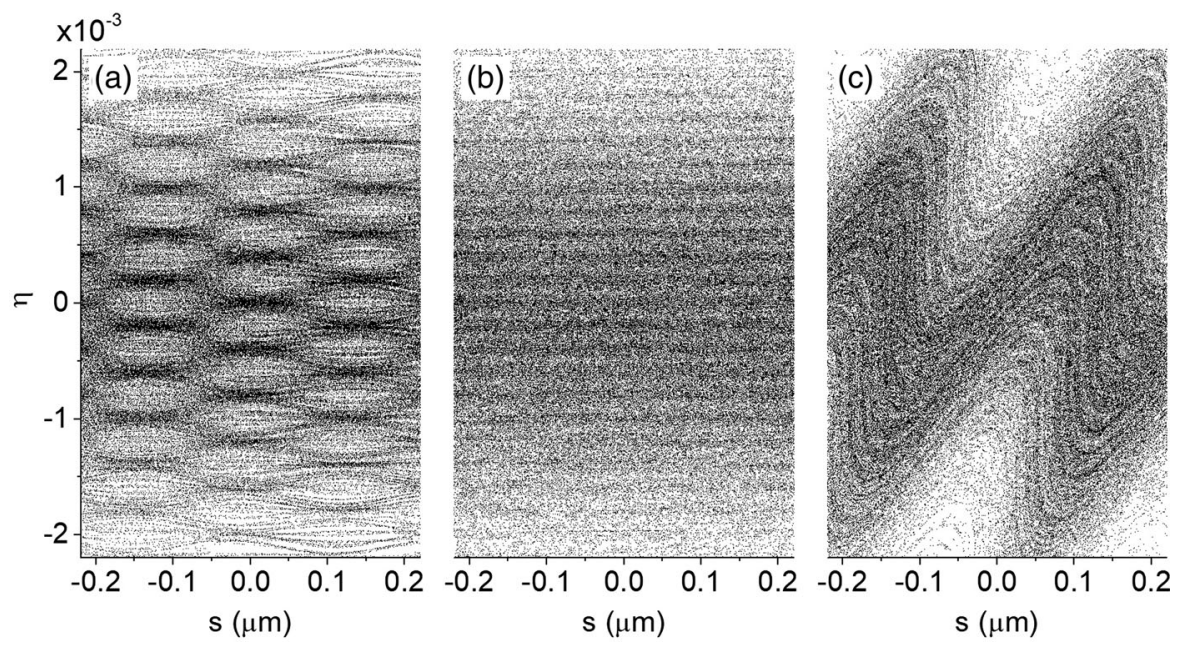

FIG. 5. Simulation results in terms of the macroparticle distributions: (a) after the first modulator as indicated by (3) in Fig. 1, (b) before the second modulator (4), and (c) before the radiator (5). 


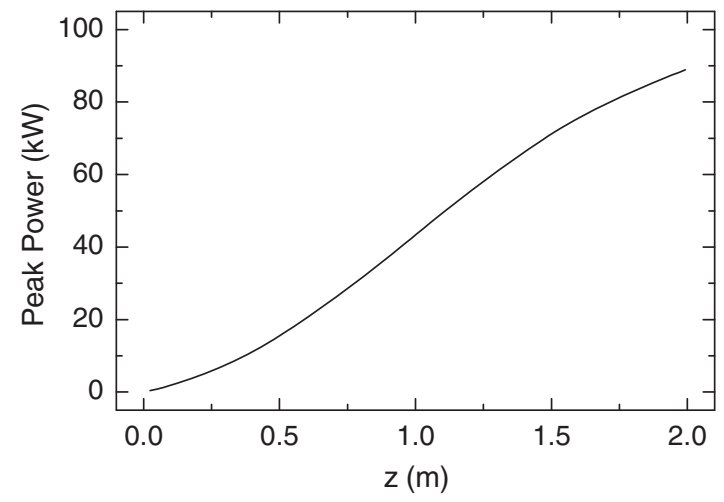

FIG. 6. Growth of the peak power at the radiator.

modulated to generate microbunches with a large harmonic order as found in (c), which is similar to the mechanism of the EEHG scheme. Note that $R_{56}$ of $0.056 \mathrm{~mm}$ is an optimum value to maximize the bunch factor at the 20th harmonic; in this example, $\left|b_{20}\right|$ is around 0.03. Although this is not a big number, it is large enough to generate intense coherent radiation in the radiator.

Figure 6 shows the growth of coherent radiation with the wavelength of $13.4 \mathrm{~nm}$, where the peak power is plotted as a function of the distance from the radiator entrance. At the exit of the radiator, the peak power approaches $100 \mathrm{~kW}$. This corresponds to the peak brightness of $6 \times 10^{29}$, if we assume that the radiation is fully coherent in time and space, and thus all the photons in the six-dimensional phase space are contained in the minimum volume defined by the Fourier and diffraction limits. This is 7 orders of magnitude higher than that of the spontaneous radiation.

To compute the time-averaged brightness, we should take into account that the electron beam quality is more or less deteriorated in the modulation process. To be specific, the energy spread increases from $0.1 \%$ to $0.11 \%$, and the vertical emittance from 0.05 to $0.065 \mathrm{~nm}$ rad in the above example. We need to wait for the electron beam to recover its equilibrium state through the radiation damping process of the SR. The emittance growth has actually an impact on the achievable performance of the proposed scheme, and thus we need to wait for $\ln (0.065 / 0.05)=0.26$ times the damping time, so that the vertical emittance recovers its equilibrium value. This is obvious from the sensitivity of the energy-band structure to the beam size in the direction of dispersion as discussed using Fig. 4(a). Assuming a typical value of a few milliseconds for the damping time [20], we need to wait for nearly $1 \mathrm{~ms}$ after the electron beam is modulated to emit coherent radiation through the proposed scheme. As a result, we can repeat the coherent radiation process using the same electron bunch 1000 times per second. Taking into account the bunch length of $50 \mathrm{ps}$ in this example, the time-averaged brightness is estimated as $6 \times 10^{29} \times 50 \times 10^{-12} \times 1000=3 \times 10^{22}$, which is 4 orders of magnitude higher than that of the spontaneous radiation.

\section{DISCUSSION}

We now compare the proposed scheme with other competing schemes, EEHG and PEHG.

Even though the requirement on the energy modulation amplitude $\eta_{M}$ is significantly relaxed in the EEHG scheme, it should be still many times larger than the initial energy spread $\sigma_{\eta}$ to achieve a high harmonic order. For example in [15], which proposes the EEHG-based coherent radiation at the wavelength of $26.7 \mathrm{~nm}$ in a SR (synchrotron SOLEIL), using the 30th harmonic of the infrared laser $(800 \mathrm{~nm})$, $\eta_{M}=5 \sigma_{\eta}$ is assumed to sufficiently modulate the electron beam, meaning that the energy spread grows by a factor of 3.7 .

In the PEHG scheme, the requirement on the energy modulation can be further relaxed, and thus the energy spread growth can be suppressed. It should be noted, however, that the energy modulation in a dispersive section gives rise to the emittance growth as in the case of the proposed scheme. Let us consider the motion of an electron passing through the PEHG system, namely the modulator and radiator located in a dispersive section, whose initial condition before entering the dispersive section is given as $x=0$ and $\eta=\eta^{\prime}$. After the dispersive section, they change as

$$
x=-\eta_{M} D \sin \left(k_{s} s\right) \eta=\eta^{\prime}+\eta_{M} \sin \left(k_{s} s\right),
$$

with which we have

$\sigma_{M}^{2}=\left\langle\int_{-\infty}^{\infty} x^{2} \delta\left(x+\eta_{M} D \sin \psi\right) d x\right\rangle=\frac{\left(\eta_{M} D\right)^{2}}{2}$.

To be specific, $\hat{\varepsilon}$ in the PEHG is roughly given as $\hat{\varepsilon}=\sqrt{1+\left(\hat{\eta_{M}} / \sqrt{2} \hat{\sigma}_{x}\right)^{2}}$, where the discrepancy in the coefficient $(\sqrt{2})$ comes from the difference in the injection scheme of the seed laser. The energy modulation required in the PEHG scheme can be evaluated by recalling that the finite beam size disturbs the microbunch formation with an effective energy spread of $\sigma_{x} / D$ [13], and thus we have $\eta_{M} \geq$ $n \sigma_{x} / D$ to reach the harmonic order of $n$, which reduces to $\hat{\varepsilon} \geq \sqrt{1+n^{2} / 2}$. For example, $\hat{\varepsilon}$ amounts to 14.2 for the 20 th harmonics.

In comparison to the above two schemes, the quality deterioration of the electron beam in the proposed scheme is expected to be much lower, because the energy modulation to be applied in the dispersive section does not have to be large; in the above example, $\eta_{M} \sim 0.1 \sigma_{\eta}$ is enough to form the pseudoenergy bands. The proposed scheme thus will offer an attractive option in SR-based light sources to significantly enhance the average brightness as well as the peak one.

Finally, let us consider how sensitive is the performance of the proposed scheme to a number of parameters, such as the energy modulation amplitude at the two modulators $\left(\eta_{M}\right.$ and $\left.\eta_{H}\right)$, intersecting angle $(\alpha)$, dispersion function $(D)$, and momentum compaction of the chicane $\left(R_{56}\right)$. If these 
parameters deviate from their design values, the bunch factor $b_{2 n}$ is given as

$$
\begin{aligned}
b_{2 n}= & \frac{\delta_{F, 1}}{2} J_{n}\left(\pi \frac{\hat{\eta}_{H}}{\hat{\eta}_{M 0}}\right) \\
& \times \exp \left[-\frac{\left(\sigma_{\eta} k_{s}\right)^{2}\left(n \delta R_{56}-2 \bar{D} \delta \alpha-2 \bar{\alpha} \delta D\right)^{2}}{2}\right],
\end{aligned}
$$

with $\bar{\alpha}$ and $\delta \alpha$ denoting the designed value of $\alpha$ and deviation from $\bar{\alpha}$, and similar expressions for other parameters.

Equation (17) allows us to roughly estimate the performance degradation of the proposed scheme due to deviations of respective parameters. Substituting the values assumed in Sec. III, we find that a deviation of $\left|\delta \eta_{M}\right| / \bar{\eta}_{M}=0.070,\left|\delta \eta_{H}\right| / \bar{\eta}_{H}=0.015, \delta \alpha=0.049 \mathrm{mrad}$, $\delta R_{56}=9.7 \times 10^{-4} \mathrm{~mm}$, or $\delta D=0.0029 \mathrm{~m}$, gives rise to a $1 \%$ reduction in $b_{2 n}$, respectively. Note that the first one regarding $\eta_{M}$ has been derived from the numerical results of $\hat{A}$ shown in Fig. 3(b) with an assumption that $\delta_{F, 1}$ is proportional to $\hat{A}$. Although it is out of scope of this paper to discuss the stability of these parameters, it is reasonable to say that development of a system to achieve the required tolerances as described above is feasible enough.

[1] A. Kondratenko and E. Saldin, Generation of coherent radiation by a relativistic electron beam in an ondulator, Part. Accel. 10, 207 (1980).

[2] L. H. Yu, Generation of intense uv radiation by subharmonically seeded single-pass free-electron lasers, Phys. Rev. A 44, 5178 (1991).

[3] P. Emma et al., First lasing and operation of an ångstromwavelength free-electron laser, Nat. Photonics 4, 641 (2010).

[4] T. Ishikawa et al., A compact X-ray free-electron laser emitting in the sub-ångström region, Nat. Photonics 6, 540 (2012).

[5] E. Allaria et al., Highly coherent and stable pulses from the FERMI seeded free-electron laser in the extreme ultraviolet, Nat. Photonics 6, 699 (2012).

[6] G. Lambert, T. Hara, D. Garzella, T. Tanikawa, M. Labat, B. Carre, H. Kitamura, T. Shintake, M. Bougeard, S. Inoue, Y. Tanaka, P. Salieres, H. Merdji, O. Chubar, O. Gobert, K. Tahara, and M.-E. Couprie, Injection of harmonics generated in gas in a free-electron laser providing intense and coherent extreme-ultraviolet light, Nat. Phys. 4, 296 (2008).

[7] T. Togashi et al., Extreme ultraviolet free electron laser seeded with high-order harmonic of Ti:sapphire laser, Opt. Express 19, 317 (2011).

[8] S. Ackermann et al., Generation of Coherent 19- and 38-nm Radiation at a Free-Electron Laser Directly Seeded at $38 \mathrm{~nm}$, Phys. Rev. Lett. 111, 114801 (2013).

[9] E. Saldin, E. Schneidmiller, and M. Yurkov, The Physics of Free Electron Lasers (Springer-Verlag, Berlin, 2000).

[10] G. Stupakov, Using the Beam-Echo Effect for Generation of Short-Wavelength Radiation, Phys. Rev. Lett. 102, 074801 (2009).

[11] D. Xiang, Z. Huang, and G. Stupakov, Generation of intense attosecond $\mathrm{X}$-ray pulses using ultraviolet laser induced microbunching in electron beams, Phys. Rev. ST Accel. Beams 12, 060701 (2009).

[12] Z. T. Zhao et al., First lasing of an echo-enabled harmonic generation free-electron laser, Nat. Photonics 6, 360 (2012).

[13] H. Deng and C. Feng, Using Off-Resonance Laser Modulation for Beam Energy Spread Cooling in Generation of Short-Wavelength Radiation, Phys. Rev. Lett. 111, 084801 (2013).

[14] C. Feng, H. Deng, D. Wang, and Z. Zhao, Phase-merging enhanced harmonic generation free-electron laser, New J. Phys. 16, 043021 (2014).

[15] C. Evain, A. Loulergue, A. Nadji, J. M. Filhol, M. E. Couprie, and A. A. Zholents, Soft X-ray femtosecond coherent undulator radiation in a storage ring, New J. Phys. 14, 023003 (2012).

[16] C. Feng, B. Jiang, Z. Qi, and Z. Zhao, Storage ring based PEHG FEL for EUV lithography, in High-Brightness Sources and Light-Driven Interactions (Optical Society of America, Long Beach, California, 2016), p. EM3A.1.

[17] M. E. Couprie, A. Loulergue, M. Labat, R. Lehe, and V. Malka, Towards a free electron laser based on laser plasma accelerators, J. Phys. B 47, 234001 (2014).

[18] T. Tanaka and H. Kitamura, SPECTRA: a synchrotron radiation calculation code, J. Synchrotron Radiat. 8, 1221 (2001).

[19] T. Tanaka, SIMPLEX: simulator and postprocessor for free-electron laser experiments, J. Synchrotron Radiat. 22, 1319 (2015).

[20] H. Wiedemann, Particle Accelerator Physics (SpringerVerlag, Berlin, 2007). 